INPLASY

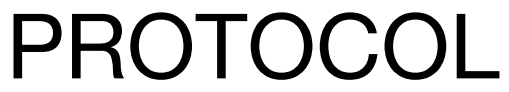

To cite: Cacciapaglia et al. Comparison of Adalimumab to other Targeted Therapies in Rheumatoid Arthritis: results from Systematic Literature review and Meta-Analysis. Inplasy protocol 202220048. doi:

10.37766/inplasy2022.2.0048

Received: 13 February 2022

Published: 13 February 2022

Corresponding author:

Fabio Cacciapaglia

fabio.cacciapaglia79@gmail.com

Author Affiliation:

Rheumatology Unit -

Department of Emergency and

Organs Transplantation (DETO);

University of Bari, Puglia, Italy.

Support: None.

Review Stage at time of this submission: Preliminary searches.

Conflicts of interest:

None declared.

\section{Comparison of Adalimumab to other Targeted Therapies in Rheumatoid Arthritis: results from Systematic Literature review and Meta-Analysis}

Cacciapaglia, F1; Venerito, V2; Stano, S3; Fornaro, M4; Lopalco, G5; lannone, $\mathrm{F}^{6}$.

Review question / Objective: To indentify which targeted therapy is more likely to achieve the $50 \%$ American College of Rheumatology (ACR50) response with good safety at 24weeks of treatment in rheumatoid arthritis (RA) patients.

Condition being studied: Head-to-head phase 3 RCTs that compared adalimumab to other targeted therapies in combination with methotrexate (MTX) or as monotherapy to treat RA patients.

Information sources: Systematic review of the literature through MEDLINE, EMBASE, Cochrane Library and Clinicaltrial.gov.

INPLASY registration number: This protocol was registered with the International Platform of Registered Systematic Review and Meta-Analysis Protocols (INPLASY) on 13 February 2022 and was last updated on 13 February 2022 (registration number INPLASY202220048).

\section{INTRODUCTION}

Review question / Objective: To indentify which targeted therapy is more likely to achieve the $50 \%$ American College of Rheumatology (ACR50) response with good safety at 24-weeks of treatment in rheumatoid arthritis (RA) patients.
Rationale: Head to head RCTs that compared biologic (b)- and targeted syntethic (ts)-DMARDs are limited to few studies and the best agents for RA treatment is unknown.

Condition being studied: Head-to-head phase 3 RCTs that compared adalimumab to other targeted therapies in combination 
with methotrexate (MTX) or as monotherapy to treat RA patients.

\section{METHODS}

Search strategy: A systematic literature review with a Bayesian network metanalysis of phase 3 RCTs, searched through MEDLINE, EMBASE, Cochrane Library and Clinicaltrial.gov.

Participant or population: Patients enrolled in phase 3 RCTs fulfilled the 2010 ACR/ EULAR classification criteria for rheumatoid arthritis.

Intervention: Adalimumab.

Comparator: Biologics or targeted synthetic DMARDs that have been compared to adalimumab.

Study designs to be included: Head-tohead phase 3 RCTsPhase 3 RCTs.

Eligibility criteria: Phase 3 RCTs that compared efficacy and safety od adalimumab to other $b$ - or ts-DMARDs in RA patients.

Information sources: Systematic review of the literature through MEDLINE, EMBASE, Cochrane Library and Clinicaltrial.gov.

Main outcome(s): The ACR50 response and withdrawals due to adverse events at 24weeks.

Quality assessment / Risk of bias analysis: The methodological qualities of all studies will be quantified using the Jadad scores. The Jadad scale assesses random assignment, double-blinding, and patient withdrawal and dropout rates. Jadad scores range from 0 to 5 . Quality was classified as high (score of 3-5) versus low (score of 0-2).

Strategy of data synthesis: Bayesian network meta-analysis (BNM) reflects a prior belief of the possible values of the model parameters of interest, whose likelihood distribution is based on the observed data, and shapes a posterior probability distribution. The reported number of patients in both study arms of every included RCT was used for each analysis. The results of this MTC will be reported as the odds ratio (OR) for a response with every single treatment evaluated head-to-head with the fixed comparator in all RCTs (adalimumab) and the OR for a response emerging from each pairwise combination of the combination treatment (b- or ts-DMARDs \pm MTX). Convergence will be verified by plots, Monte Carlo error monitoring and with the support of Gelman-Rubin diagnostics, reached at n.100'000 iterations. The pairwise odds ratio (OR) and $95 \%$ credible interval (Crl) (or Bayesian $\mathrm{Cl}$ ) and adjusted for multiple-arm trials will be reported. Information about the relative effects will be converted to a probability and then a ranking of each treatment will be presented according to the surface under the cumulative ranking curve (SUCRA), expressed a percentage - the SUCRA would be $100 \%$ when a treatment is certain to be the best and $0 \%$ when a treatment is certain to be the worst.

Subgroup analysis: The WinBUGS 1.4 software (MRC Biostatistics Unit, Cambridge, UK) will be used to combine the direct and indirect evidence of this Bayesian model for the present network meta-analysis.

Sensitivity analysis: Considering the presumed small number of included studies in the individual arms and to avoid the influence of heterogeneity in demographic characteristics of patients included across the different RCTs with different disease duration and ethnicity, we will use a random-effects model for this network meta-analysis. This method is more appropriate to detect a small real difference and minimize the interference of sample size variability among different studies analysed, with patients from each part of the world.

Language: English.

Country(ies) involved: Italy. 
Keywords: Efficacy; Safety; Bayesian Metaanalysis; Rheumatoid Arthritis; Targeted Therapies.

Dissemination plans: Publication on an international preer-review Journal.

Contributions of each author:

Author 1 - Fabio Cacciapaglia - Author 1 conceived the study, will perfome the revision of the literature and draft the manuscript.

Email: fabio.cacciapaglia79@gmail.com

Author 2 - Vincenzo Venerito - Author 2 conceived the study, will perfome the revision of the literature and statistical analysis.

Email: vincenzo.venerito@gmail.com

Author 3 - Stefano Stano - Author 3 will perfome the revision of the literature.

Author 4 - Marco Fornaro - Author 4 will collect the data and collaborate to statistical analysis.

Email: marco.fornaro@uniba.it

Author 5 - Giuseppe Lopalco - Author 5 will collect the data and contribute to data analysis.

Email: giuseppe.lopalco@uniba.it

Author 6 - Florenzo lannone - Author 6 conceived and designed the analysis and will contribute to wrote the final manuscript.

Email: florenzo.iannone@uniba.it 